\title{
LV.
}

\section{Ueber eine neue Art der Bildung des Aethylens und einiger verwandter Verbindungen.}

\section{Von}

\section{A. Boutlerow.}

(Compt. rend. t. LIII, p. 247.)

Als ich versuchte, das Aethylen durch Zersetzung des Jodmethylens $\mathbf{C H}_{2} \mathrm{~J}_{2}$ mittelst Natriumamalgam oder anderer Metalle $\mathbf{z u}$ isoliren, erhielt ich Resultate, welche von Interesse zu sein scheinen.

Wenn man Methylenjodür mit Kupfer und Wasser bei $100^{\circ}$ in zugeschmolzener Röhre erhitzt, so bildet sich Kupferjodür und es entwickelt sich ein Gemenge von Kohlensäure, Kohlenoxyd, Sumpfgas und ein Gemisch der Kohlenwasserstoffe $\in_{\mathrm{n}} \mathrm{H}_{2 \mathrm{n}}$. Das Gas wurde durch Kali von $\mathrm{K}_{\mathrm{oh}}$ lensäure befreit und dann mit Brom behandelt; hierbei verschwanden 85 Volproc. und es bildete sich eine ölige Flüssigkeit, ein Gemenge von Bromüren, die fractionirt destillirt wurden. Der grösste Theil der Flüssigkeit ging bei 131 bis $132^{\circ}$ über, und der Siedepunkt stieg endlich bis $180^{\circ}$ und selbst darüber.

Das bei $131-132^{\circ}$ übergehende Product war reines Aethylenbromür von 2,179 spec. Gew. Der wenigst flüchtige Theil war ein Gemenge von Aethylenbromür mit zusammengesetzteren Bromüren $\boldsymbol{\epsilon}_{\mathrm{n}} \mathrm{H}_{2 \mathrm{n}} \mathrm{Br}_{2}$.

Aus diesen Versuchen geht hervor, dass sich bei Einwirkung von Kupfer und Wasser aut Methylenjodür, $\mathbf{C H}_{2} \mathrm{~J}_{2}$, kein Aethylen bildet. Das Molekül $\mathrm{CH}_{2}$, welches in Freiheit gesetzt wird, verdoppelt sich, um Aethylen und höhere Kohlenwasserstoffe zu bilden. Auch ist es nach diesen Versuchen zweifelhaft, ob Aethylen im freien Zustande existi ren kann. Das Aethylen und seine höheren Homologen sind damit polymer. In allen Fällen ist die beschriebenc 
Thatsache ein interessantes Beispiel molekularer Complication, hervorgebracht auf synthetischem Wege. Indem man das Jodmethylen in Aethylen umwandelt, verdoppelt man das Molekül seines Radicals, und kann mit dem so entstandenen Aethylen Alkohol, die erste Quelle für die Methylenverbindungen, regeneriren.

\section{LVI. \\ Ueber die Oxäthylenbasen.}

Von

Ad. Würtz.

(Compt. rend. t. LIII, p. 338.)

Ich habe vor einiger Zeit*) gezeigt, dass sich das Aethylenoxyd direct mit Ammoniak verbindet und damit samerstoffhaltige Basen bildet, welche nach Art ihrer Entstehung und ihrer Molekularstructur weit von den eigentlichen zusammengesetzten Ammoniaken abweichen. A. a. O. hake ich die Existenz zweier solcher Basen beschrieben; die erste durch Verbindung von $2 \mathrm{Mol}$. Aethylenoxyd mit 1 Mol. Ammoniak, die zweite aus 3 Mol. Aethylenoxyd und $1 \mathrm{Mol}$. Ammoniak entstehend. Nunmehr habe ich diese Reihe vervollständigt.

Verdampft man das Product der Reaction von Ammoniak auf Aethylenoxyd im Wasserbade und sättigt den syrupdicken Rückstand mit Chlorwasserstoffsäure, so erhält man, wie ich zeigte, ein Gemenge von Hydrochloraten, welche man durch absoluten Alkohol trennen kann. Das eine von diesen, das Chlorwasserstoff-Trioxäthylenamin, $\left[\left(-\mathrm{C}_{2} \mathrm{H}_{4} \Theta\right) \mathrm{NH}_{3}\right] \mathrm{HCl}$, ist unlöslich. Aus der alkoholischen Lösung habe ich als Platindoppelsalz die Verbindung der Base Dioxäthylen gefällt: $\left[\left(\mathrm{G}_{2} \mathbf{H}_{4} \boldsymbol{\Theta}\right)_{2} \mathrm{NH}_{3}\right] \mathrm{HCl}, \mathrm{PtCl}_{2}$.

-) Dies. Journ. LXXXI, 94. 